\title{
A Generalized Mazur-Ulam Theorem for Fuzzy Normed Spaces
}

\author{
J. J. Font, J. Galindo, S. Macario, and M. Sanchis \\ Departament de Matemàtiques, Institut Universitari de Matemàtiques i Aplicacions de Castelló (IMAC), Universitat Jaume I, \\ Campus del Riu Sec. s/n, 12071 Castelló, Spain \\ Correspondence should be addressed to S. Macario; macario@mat.uji.es
}

Received 3 April 2014; Accepted 19 May 2014; Published 2 June 2014

Academic Editor: Salvador Romaguera

Copyright (C) 2014 J. J. Font et al. This is an open access article distributed under the Creative Commons Attribution License, which permits unrestricted use, distribution, and reproduction in any medium, provided the original work is properly cited.

We introduce fuzzy norm-preserving maps, which generalize the concept of fuzzy isometry. Based on the ideas from Vogt, 1973, and Väisälä, 2003, we provide the following generalized version of the Mazur-Ulam theorem in the fuzzy context: let $X, Y$ be fuzzy normed spaces and let $f: X \rightarrow Y$ be a fuzzy norm-preserving surjection satisfying $f(0)=0$. Then $f$ is additive.

\section{Introduction}

Studies on fuzzy normed spaces are relatively recent in the field of fuzzy functional analysis. It was Katsaras who in 1984 [1], while studying topological vector spaces, was the first to introduce the idea of fuzzy norm on a linear space. Eight years later, Felbin [2] offered an alternative definition. With this definition, the induced fuzzy metric is of Kaleva and Seikkala type [3]. In 1994, Cheng and Mordeson [4] defined another type of fuzzy norm on a linear space whose associated fuzzy metric is of Kramosil and Michalek type [5]. Finally, in [6] (see also [7]), Bag and Samanta redefined the concept of fuzzy norm given in [4] as follows.

Definition 1. Let $X$ be a real linear space. A function $N: X \times$ $\mathbb{R} \rightarrow[0,1]$ is said to be a fuzzy norm on $X$ if, for all $x, y \in X$ and all $t, s, k \in \mathbb{R}$, it satisfies the following:

(N1) $N(x, t)=0$ for $t \leq 0$;

(N2) $x=0$ if and only if $N(x, t)=1$ for all $t>0$;

(N3) $N(k x, t)=N(x, t /|k|)$ if $k \neq 0$;

(N4) $N(x+y, t+s) \geq \min \{N(x, t), N(y, s)\}$;

(N5) $\lim _{t \rightarrow \infty} N(x, t)=1$.

The pair $(X, N)$ is called a fuzzy normed space.
We point out that classical normed spaces are strictly included in the class of fuzzy normed spaces (see [6]) and that (N2) and (N4) imply that, for a fixed $x \in X$, the function $N(x, \cdot)$ is nondecreasing. It is a well-known fact that every fuzzy norm on a real linear space $X$ induces a topology on $X$ defined as follows: a subbase for the neighborhood system at a point $x \in X$ consists of the sets

$$
B_{N}(x, \varepsilon, t)=\{y \in X: N(x-y, t)>1-\varepsilon\}
$$

for all $0<\varepsilon<1$ and $t>0$. It is straightforward to verify that the filter of neighborhoods of the origin generated by the family $\left\{B_{N}(0, \varepsilon, t): 0<\varepsilon<1, t>0\right\}$ satisfies the properties which make $(X, N)$ a Hausdorff topological vector space.

The theory of isometric mappings on classical normed spaces has its roots in the seminal paper by Mazur and Ulam ([8]; see also [9]), who proved that every bijective isometry between two real normed spaces is affine. It is known that the surjective assumption is essential in this result and that it is not true for complex normed spaces. The MazurUlam theorem has been extended in many directions. For example, Baker [10] proved that the result remains true if we consider an (not necessarily onto) isometry between a real normed space and a strictly convex real normed space. Another direction was provided by Vogt [11], who replaced isometries by the more general notion of equality of distance preserving maps (see also [12]). 
In this paper, following the ideas of Vogt, we introduce a generalization of the concept of fuzzy isometry as follows.

Definition 2. Let $(X, N)$ and $\left(Y, N^{\prime}\right)$ be two fuzzy normed spaces. One says that $f: X \rightarrow Y$ is a fuzzy norm-preserving mapping if given $x, y, x^{\prime}, y^{\prime} \in X$, then, for all $t>0$,

$$
\begin{aligned}
N(x-y, t) & =N\left(x^{\prime}-y^{\prime}, t\right) \Longrightarrow N^{\prime}(f(x)-f(y), t) \\
& =N^{\prime}\left(f\left(x^{\prime}\right)-f\left(y^{\prime}\right), t\right) .
\end{aligned}
$$

Let us recall here the definition of a fuzzy isometry.

Definition 3. Let $(X, N)$ and $\left(Y, N^{\prime}\right)$ be two fuzzy normed spaces. It is said that $f: X \rightarrow Y$ is a fuzzy isometry if $N^{\prime}(f(x)-f(y), t)=N(x-y, t)$, for all $x, y \in X$ and $t>0$.

We provide the following generalized version of the Mazur-Ulam theorem in the fuzzy context: let $X, Y$ be fuzzy normed spaces and let $f: X \rightarrow Y$ be a fuzzy normpreserving surjection satisfying $f(0)=0$. Then, $f$ is additive. As a corollary, we deduce that if such an $f$ is a fuzzy isometry, then $f$ is affine.

\section{The Results}

Let $(X, N)$ be a fuzzy normed space. Fix $c \in X$ and define a function $\phi_{c}: X \rightarrow X$ as $\phi_{c}(x):=2 c-x$. It is apparent that $\phi_{c}$ is bijective; indeed, $\phi_{c} \circ \phi_{c}=I d$, where $I d$ stands for the identity map on $X$. Furthermore, $\phi_{c}$ is a fuzzy isometry since $N\left(\phi_{c}(x)-\phi_{c}(y), t\right)=N(2 c-x-2 c+y, t)=N(x-y, t)$, for all $x, y \in X$ and $t>0$.

Let $L(c, N):=\{x \in X: N(x, t)=N(2 c-x, t) \geq$ $N(c, t / 2)$ for all $t>0\}$. It is clear that $c \in L(c, N) \neq \emptyset$ and that $\phi_{c}(L(c, N)) \subseteq L(c, N)$.

Lemma 4. Every fuzzy isometry from $L(c, N)$ onto $L(c, N)$ fixes $c$.

Proof. Let $I \quad:=\{g \quad: \quad L(c, N) \rightarrow L(c, N) \quad \rightarrow$ $g$ is an onto fuzzy isometry\}. This is a nonempty set since the identity map belongs to it. Fix $t>0$ and let us define, for each $i=1,2,3, \ldots$,

$$
\lambda_{i}^{t}:=\inf \left\{N\left(g(c)-c, \frac{t}{i}\right): g \in I\right\} .
$$

If $g \in I$, then we define $g^{\prime}:=g^{-1} \circ \phi_{c} \circ g$, which is in $I$. Then

$$
\begin{aligned}
N\left(g^{\prime}(c)-c, t\right) & =N\left(\left(g^{-1} \circ \phi_{c} \circ g\right)(c)-c, t\right) \\
& =N\left(\phi_{c}(g(c))-g(c), t\right) \\
& =N(2(g(c)-c), t) \\
& =N\left(g(c)-c, \frac{t}{2}\right) .
\end{aligned}
$$

Consequently, $N(g(c)-c, t / 2) \geq \lambda_{1}^{t}$.

Moreover, for all $g \in I$, we have

$$
N(g(c)-c, t) \geq N\left(g(c)-c, \frac{t}{2}\right) \geq \lambda_{1}^{t},
$$

which yields $\lambda_{1}^{t} \geq \lambda_{2}^{t} \geq \lambda_{1}^{t}$. That is, $\lambda_{1}^{t}=\lambda_{2}^{t}$, which leads us to the following equalities:

$$
\lambda_{1}^{t}=\lambda_{2}^{t}=\cdots=\lambda_{n}^{t}=\cdots .
$$

On the other hand, given $g \in I$, we have

$$
\begin{aligned}
N\left(c, \frac{t}{2}\right) \leq \lambda_{n_{t}}^{t} & \leq N\left(g_{0}(c)-c, \frac{t}{n_{t}}\right) \leq N\left(g_{0}(c)-c, t_{0}\right) \\
& =k<1,
\end{aligned}
$$

since $g(c) \in L(c, N)$.

Let us suppose that there exists $g_{0} \in I$ such that $g_{0}(c) \neq c$. Then, there exists $t_{0}>0$ such that

$$
N\left(g_{0}(c)-c, t_{0}\right)=k<1 .
$$

In addition, for each $t>0$, there exists $n_{t}$ such that $N\left(g_{0}(c)-\right.$ $\left.c, t / n_{t}\right) \leq N\left(g_{0}(c)-c, t_{0}\right)$. Hence, by (6) and (7), we have that

$$
\begin{aligned}
N\left(c, \frac{t}{2}\right) & \leq \lambda_{n_{t}}^{t} \leq N\left(g_{0}(c)-c, \frac{t}{n_{t}}\right) \leq N\left(g_{0}(c)-c, t_{0}\right) \\
= & k<1,
\end{aligned}
$$

but

$$
1=\lim _{t \rightarrow \infty} N\left(c, \frac{t}{2}\right) \leq k<1,
$$

a contradiction which completes the proof.

Remark 5. It can be checked that a fuzzy norm-preserving mapping is associated, for each $t>0$, with a function $\rho_{t}$ : $D_{t} \subseteq[0,1] \rightarrow[0,1]$ such that

$$
N^{\prime}(f(x)-f(y), t)=\rho_{t}(N(x-y, t)) .
$$

It is then apparent that fuzzy isometries are fuzzy normpreserving mappings taking $\rho_{t}=I d, t>0$.

Theorem 6. Let $(X, N)$ and $\left(Y, N^{\prime}\right)$ be two fuzzy normed spaces and let $f: X \rightarrow Y$ be a fuzzy norm-preserving surjection satisfying $f(0)=0$. Then $f$ is additive.

Proof. Fix $x_{0} \in X$ and let

$$
\begin{array}{r}
L\left(f\left(x_{0}\right), N^{\prime}\right) \\
\quad:=\left\{y \in Y: N^{\prime}(y, t)=N^{\prime}\left(2 f\left(x_{0}\right)-y, t\right)\right. \\
\left.\quad \geq N^{\prime}\left(f\left(x_{0}\right), \frac{t}{2}\right), t>0\right\} .
\end{array}
$$

We know that $f\left(x_{0}\right) \in L\left(f\left(x_{0}\right), N^{\prime}\right) \neq \emptyset$.

We now define map $h: L\left(f\left(x_{0}\right), N^{\prime}\right) \rightarrow L\left(f\left(x_{0}\right), N^{\prime}\right)$ as $h(y):=f\left(x_{1}-x^{\prime}\right)$ for a fixed $x_{1} \in f^{-1}\left(2 f\left(x_{0}\right)\right)$ and $x^{\prime} \in$ $f^{-1}(y)$. 
Claim $1 . h$ is a fuzzy isometry from $L\left(f\left(x_{0}\right), N^{\prime}\right)$ to $L\left(f\left(x_{0}\right), N^{\prime}\right)$ which does not depend on the choice of $x^{\prime} \in$ $f^{-1}(y)$.

Let us first check that $h$ does not depend on the choice of $x^{\prime} \in f^{-1}(y)$. To this end, suppose that $\left\{x^{\prime}, x^{\prime \prime}\right\} \subseteq f^{-1}(y)$. Then,

$$
\begin{aligned}
N^{\prime}( & \left.f\left(x_{1}-x^{\prime}\right)-f\left(x_{1}-x^{\prime \prime}\right), t\right) \\
\quad & \rho_{t}\left(N\left(x^{\prime \prime}-x^{\prime}, t\right)\right)=N^{\prime}\left(f\left(x^{\prime \prime}\right)-f\left(x^{\prime}\right), t\right) \\
& =N^{\prime}(0, t)=1,
\end{aligned}
$$

which is to say that $f\left(x_{1}-x^{\prime}\right)=f\left(x_{1}-x^{\prime \prime}\right)$.

Let us next prove that $h$ is a fuzzy isometry. Suppose that $f\left(x^{\prime}\right)=y^{\prime}$ and $f\left(x^{\prime \prime}\right)=y^{\prime \prime}$, with $x^{\prime}, x^{\prime \prime} \in X$ and $y^{\prime}, y^{\prime \prime} \in Y$. Then,

$$
\begin{aligned}
N^{\prime}\left(h\left(y^{\prime}\right)-h\left(y^{\prime \prime}\right), t\right) & =N^{\prime}\left(f\left(x_{1}-x^{\prime}\right)-f\left(x_{1}-x^{\prime \prime}\right), t\right) \\
& =\rho_{t}\left(N\left(x^{\prime \prime}-x^{\prime}, t\right)\right) \\
& =N^{\prime}\left(f\left(x^{\prime \prime}\right)-f\left(x^{\prime}\right), t\right) \\
& =N^{\prime}\left(y^{\prime \prime}-y^{\prime}, t\right) .
\end{aligned}
$$

Finally, let us check that $h$ maps $L\left(f\left(x_{0}\right), N^{\prime}\right)$ onto $L\left(f\left(x_{0}\right), N^{\prime}\right)$. Fix $y^{\prime} \in L\left(f\left(x_{0}\right), N^{\prime}\right)$ and let $x^{\prime} \in X$ such that $f\left(x^{\prime}\right)=y^{\prime}$. From the definition of $L\left(f\left(x_{0}\right), N^{\prime}\right)$, we know that

$$
N^{\prime}\left(y^{\prime}, t\right)=N^{\prime}\left(2 f\left(x_{0}\right)-y^{\prime}, t\right) \geq N^{\prime}\left(f\left(x_{0}\right), \frac{t}{2}\right) .
$$

Hence,

$$
\begin{aligned}
N^{\prime}\left(h\left(y^{\prime}\right), t\right) & =N^{\prime}\left(f\left(x_{1}-x^{\prime}\right)-f(0), t\right) \\
& =\rho_{t}\left(N\left(x_{1}-x^{\prime}-0, t\right)\right) \\
& =N^{\prime}\left(f\left(x_{1}\right)-f\left(x^{\prime}\right), t\right) \\
& =N^{\prime}\left(2 f\left(x_{0}\right)-y^{\prime}, t\right) \\
& =N^{\prime}\left(y^{\prime}, t\right) \\
& \geq N^{\prime}\left(f\left(x_{0}\right), \frac{t}{2}\right) .
\end{aligned}
$$

Furthermore,

$$
\begin{aligned}
N^{\prime}\left(2 f\left(x_{0}\right)-h\left(y^{\prime}\right), t\right) & =N^{\prime}\left(2 f\left(x_{0}\right)-f\left(x_{1}-x^{\prime}\right), t\right) \\
& =N^{\prime}\left(f\left(x_{1}\right)-f\left(x_{1}-x^{\prime}\right), t\right) \\
& =\rho_{t}\left(N\left(x_{1}-x_{1}+x^{\prime}-0, t\right)\right) \\
& =N^{\prime}\left(f\left(x^{\prime}\right)-f(0), t\right) \\
& =N^{\prime}\left(y^{\prime}, t\right) \\
& =N^{\prime}\left(h\left(y^{\prime}\right), t\right) .
\end{aligned}
$$

As a consequence, we deduce that $h\left(L\left(f\left(x_{0}\right), N^{\prime}\right)\right) \subseteq$ $L\left(f\left(x_{0}\right), N^{\prime}\right)$. Since it is a routine matter to verify that $h^{-1}=h$, we infer that $h\left(L\left(f\left(x_{0}\right), N^{\prime}\right)\right)=L\left(f\left(x_{0}\right), N^{\prime}\right)$, and the claim is proved.

Thanks to Claim 1, we can apply Lemma 4 and conclude that $h$ fixes $f\left(x_{0}\right)$. Hence,

$$
f\left(x_{0}\right)=h\left(f\left(x_{0}\right)\right)=f\left(x_{1}-x_{0}\right)
$$

and, then,

$$
\begin{aligned}
1 & =N^{\prime}\left(f\left(x_{0}\right)-f\left(x_{1}-x_{0}\right), t\right) \\
& =\rho_{t}\left(N\left(2 x_{0}-x_{1}, t\right)\right) \\
& =N^{\prime}\left(f\left(2 x_{0}\right)-f\left(x_{1}\right), t\right) \\
& =N^{\prime}\left(f\left(2 x_{0}\right)-2 f\left(x_{0}\right), t\right)
\end{aligned}
$$

for all $t>0$, which is to say that

$$
f\left(2 x_{0}\right)=2 f\left(x_{0}\right) .
$$

Next, let us define, for a fixed $z \in X$, the following map:

$$
f_{z}(x):=f(x+z)-f(z),
$$

for all $x \in X$.

It is clear that $f_{z}(0)=0$ and $f_{z}$ is surjective since $f$ is assumed to be also surjective. Furthermore, for all $x, y \in X$ and $t>0$,

$$
\begin{aligned}
& N^{\prime}\left(f_{z}(x)-f_{z}(y), t\right) \\
& \quad=N^{\prime}(f(x+z)-f(y+z), t)=\rho_{t}(N(x-y, t)),
\end{aligned}
$$

which is to say that $f_{z}$ is also a fuzzy norm-preserving map. Hence, by (20), we infer that $f_{z}(2 x)=2 f_{z}(x)$, for all $x \in X$. Then, for all $x, y \in X$, we have

$$
\begin{aligned}
& f((x-y)+y)-f(y) \\
& \quad=f_{y}(x-y)=2 f_{y}\left(\frac{x-y}{2}\right) \\
& \quad=2\left(f\left(\frac{x-y}{2}+y\right)-f(y)\right),
\end{aligned}
$$


which yields

$$
f(x)+f(y)=2 f\left(\frac{x+y}{2}\right)=f(x+y) ;
$$

that is, $f$ is additive.

Let us recall here that additivity yields $\mathbb{Q}$-linearity, which, in presence of continuity, implies linearity. Hence, as a straightforward corollary of Theorem 6, we obtain a fuzzy version of the Mazur-Ulam theorem.

Corollary 7. Let $(X, N)$ and $\left(Y, N^{\prime}\right)$ be two fuzzy normed spaces and let $f: X \rightarrow Y$ be a surjective fuzzy isometry. Then, $f$ is affine.

Proof. It suffices to apply Theorem 6 to $g(x):=f(x)-f(0)$, $x \in X$.

\section{Conflict of Interests}

The authors declare that there is no conflict of interests regarding the publication of this paper.

\section{Acknowledgments}

This research is supported by the Spanish Ministry of Education and Science (Grant no. MTM2011-23118) and by Bancaixa (Project P1-1B2011-30).

\section{References}

[1] A. K. Katsaras, "Fuzzy topological vector spaces. II," Fuzzy Sets and Systems, vol. 12, no. 2, pp. 143-154, 1984.

[2] C. Felbin, "Finite-dimensional fuzzy normed linear space," Fuzzy Sets and Systems, vol. 48, no. 2, pp. 239-248, 1992.

[3] O. Kaleva and S. Seikkala, "On fuzzy metric spaces," Fuzzy Sets and Systems, vol. 12, no. 3, pp. 215-229, 1984.

[4] S. C. Cheng and J. N. Mordeson, "Fuzzy linear operators and fuzzy normed linear spaces," Bulletin of the Calcutta Mathematical Society, vol. 86, no. 5, pp. 429-436, 1994.

[5] I. Kramosil and J. Michálek, "Fuzzy metrics and statistical metric spaces," Kybernetika, vol. 11, no. 5, pp. 326-334, 1975.

[6] T. Bag and S. K. Samanta, "Finite dimensional fuzzy normed linear spaces," Journal of Fuzzy Mathematics, vol. 11, no. 3, pp. 687-705, 2003.

[7] T. Bag and S. K. Samanta, "Fuzzy bounded linear operators," Fuzzy Sets and Systems, vol. 151, no. 3, pp. 513-547, 2005.

[8] S. Mazur and S. Ulam, "Sur les transformations isometriques d'espaces vectoriels normes," Comptes Rendus de l'Académie des Sciences, vol. 194, pp. 946-948, 1932.

[9] J. Väisälä, "A proof of the Mazur-Ulam theorem," The American Mathematical Monthly, vol. 110, no. 7, pp. 633-635, 2003.

[10] J. A. Baker, "Isometries in normed spaces," The American Mathematical Monthly, vol. 78, pp. 655-658, 1971.

[11] A. Vogt, "Maps which preserve equality of distance," Studia Mathematica, vol. 45, pp. 43-48, 1973.

[12] R. Hu, "On the maps preserving the equality of distance," Journal of Mathematical Analysis and Applications, vol. 343, no. 2, pp. 1161-1165, 2008. 


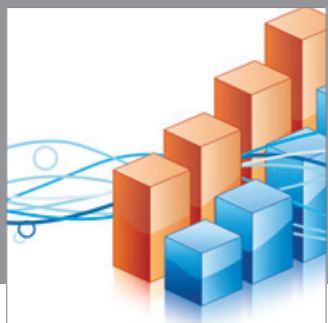

Advances in

Operations Research

mansans

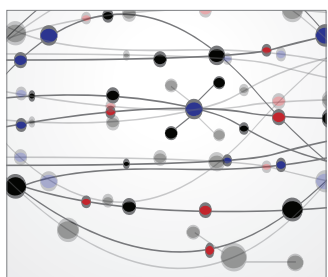

The Scientific World Journal
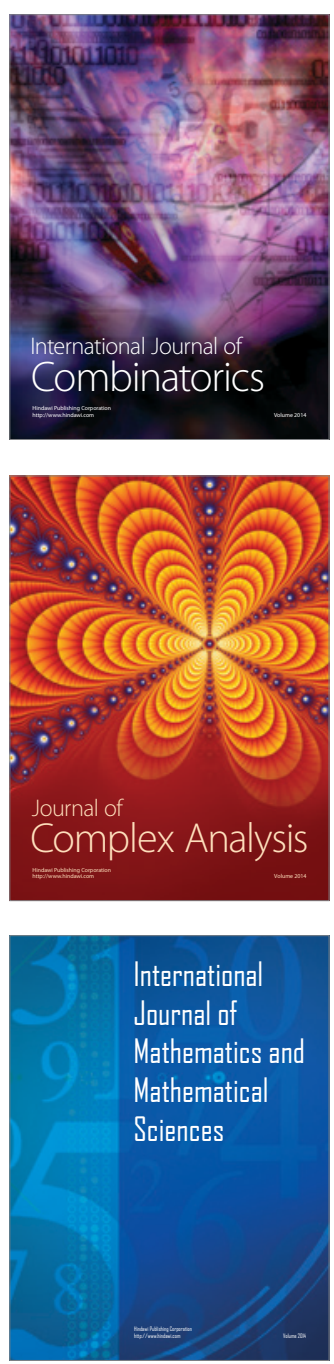
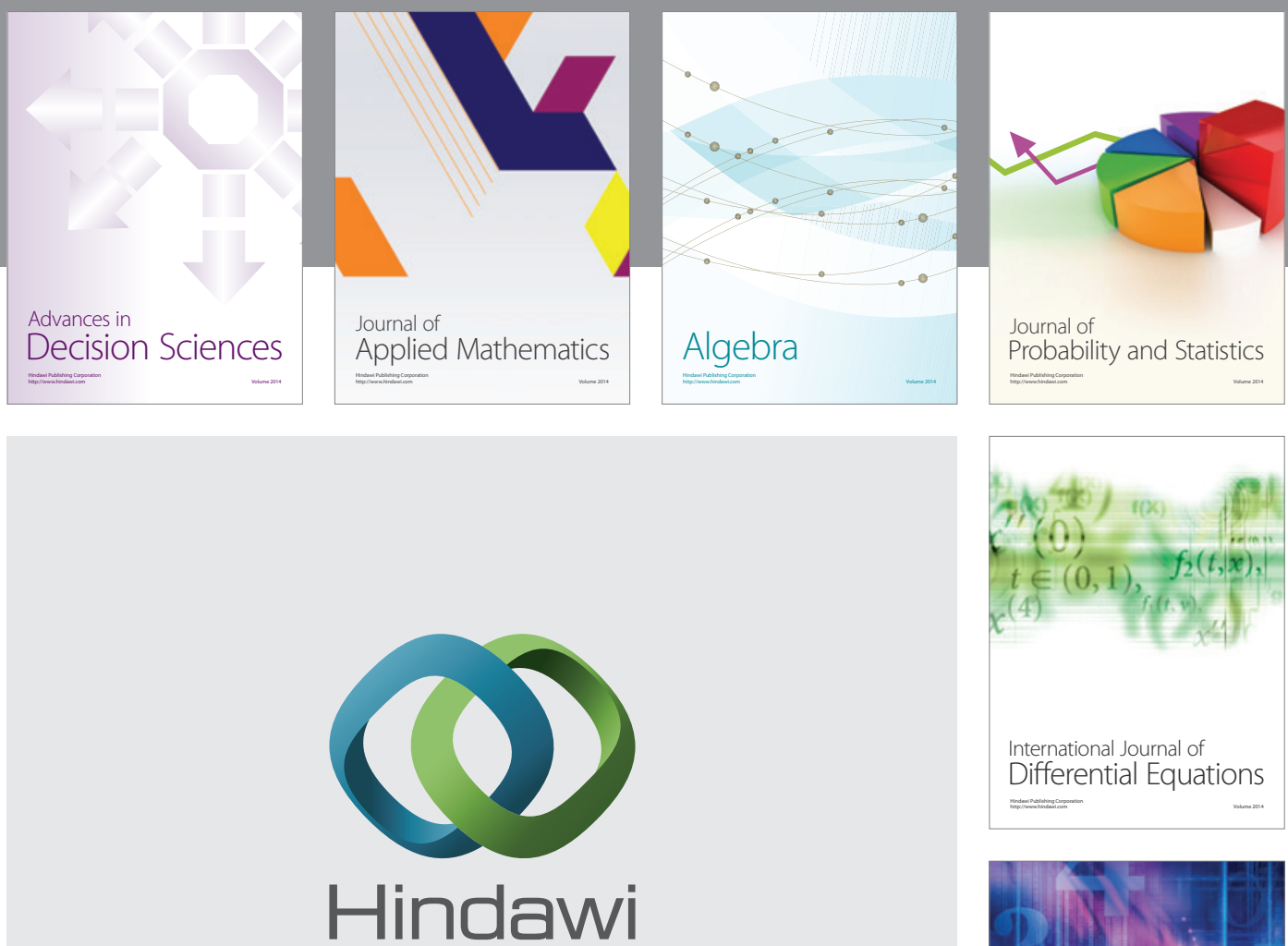

Submit your manuscripts at http://www.hindawi.com
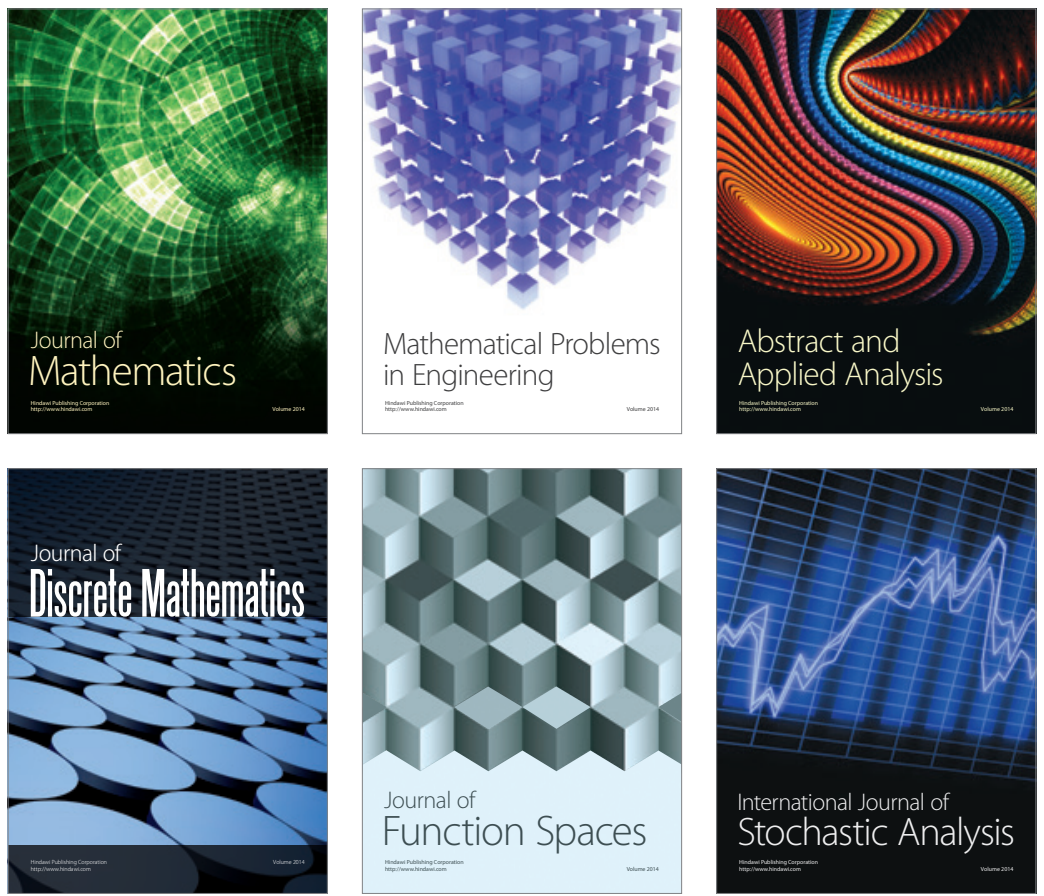

Journal of

Function Spaces

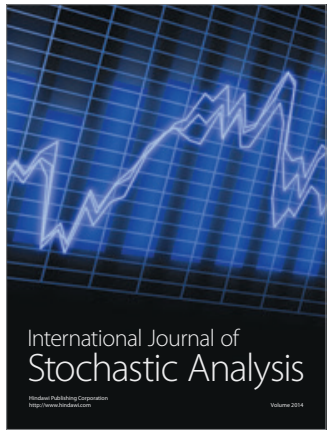

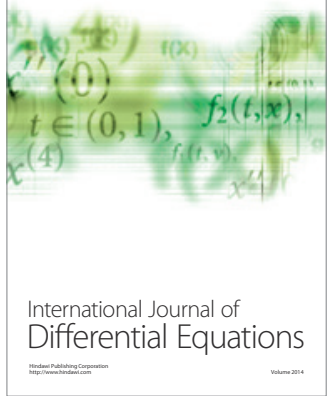
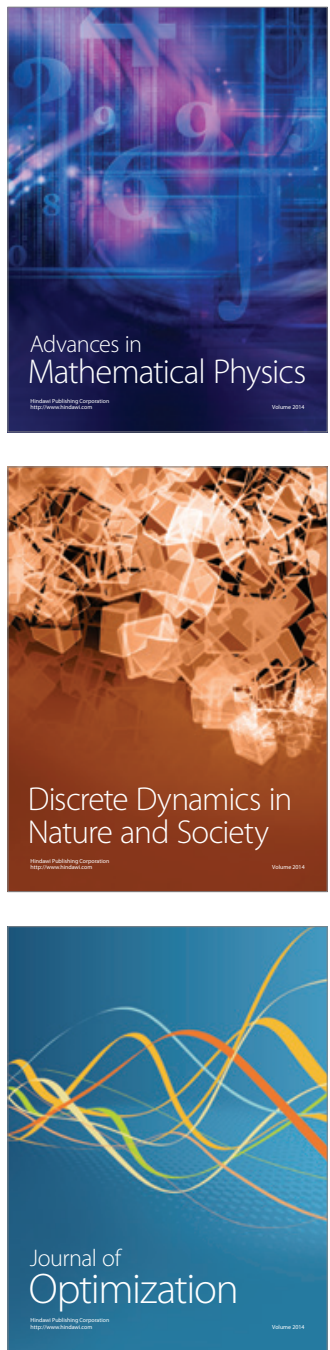